\title{
Complications of Regional Citrate Anticoagulation for Continuous Renal Replacement Therapy: An Observational Study
}

\author{
Nathan Axel Bianchi ${ }^{b}$ Marco Altarelli ${ }^{a} \quad$ Philippe Eckert ${ }^{a}$ b \\ Antoine Guillaume Schneider ${ }^{a}$ b \\ ${ }^{a}$ Adult Intensive Care Unit, Centre Hospitalier Universitaire Vaudois, Lausanne, Switzerland; \\ ${ }^{b}$ Faculty of Biology and Medicine, University of Lausanne, Lausanne, Switzerland
}

\section{Keywords}

Continuous renal replacement therapy · Regional citrate anticoagulation · Metabolic complications · Dialysis · Renal replacement therapy · Acute kidney failure

\begin{abstract}
Introduction: Regional citrate anticoagulation (RCA) is the recommended anticoagulation modality for continuous renal replacement therapy (CRRT). RCA was associated with a low rate of complications in randomized controlled trials. However, little is known about the type and rate of complications in real life. We sought to describe complications associated with RCA in comparison with those associated with heparin anticoagulation. Methods: In our institution, RCA has been the default anticoagulation modality for CRRT in all patients without contraindications since 2013 . We have retrospectively reviewed all consecutive patients who received CRRT between January and December 2016 in our institution. For each CRRT session, we have assessed circuit duration, administered dose, as well as therapy-associated complications. Those parameters were compared according to whether the circuit was run in continuous veno-venous hemodialysis (CVVHD) mode with RCA or continuous veno-venous hemofiltration (CVVH) mode with heparin anticoagulation. Results: We analyzed 691 CRRT sessions in 121 patients. Of those 400 (57.9\%) were performed in CVVHD-RCA mode
\end{abstract}

and 291 (42.1\%) in CVVH-Heparin Mode. Compared with CVVH-Heparin mode, CVVHD-RCA mode was associated with a longer circuit lifespan (median duration 54.9 interquartile range [IQR 44.6] vs. $15.3 \mathrm{~h}$ [IQR 22.4], $p<0.0001$ ). It was associated with a higher rate of metabolic acidosis $77(20.2 \%)$ vs. $18(7.2 \%),(p<0.0001)$, alkalosis $186(48.7 \%)$ vs. $43(17.1 \%)$, $(p=0.0001)$, and hypocalcemia $96(25.07 \%)$ vs. 26 events $(10.79 \%), p<0.0001$. However, the majority of these alterations were of benign or moderate severity. Only one possible citrate intoxication was observed. Conclusions: CVVHD-RCA was associated with a much longer circuit life but an increased rate of minor metabolic complications, in particular acid-base derangements. Some of these complications might have been prevented by therapy adaptation. Medical and nursing staff education is of major importance in the implementation of an RCA protocol. @ 2020 The Author(s)

Published by S. Karger AG, Basel

\section{Introduction}

Regional citrate anticoagulation (RCA) is the recommended anticoagulation strategy for continuous renal replacement therapy (CRRT) in patients without contraindications [1]. The safety of RCA protocols has largely been addressed in many clinical conditions in several ran-

Dr. Antoine Guillaume Schneider 
domized controlled trials [2-4]. Together, these studies have demonstrated RCA superiority over systemic heparin anticoagulation in terms of filter lifespan and bleeding complications without increasing the rate of metabolic alkalosis [5]. Large, observational studies from centers with extensive experience with RCA [6] confirm these results and report a very low rate of metabolic complications and citrate intoxications in unselected patients undergoing CRRT. RCA might even be safe in patients with severe liver failure [7].

Despite these compelling evidence and the seemingly obvious benefit of the modality, its acceptance and utilization remain relatively low throughout the world. Indeed, in a sub-analysis of the international observational Do-REMI-FA study [8], RCA was utilized in $<20 \%$ of circuits, while no anticoagulation at all was still frequently prescribed. This seems like a minor increase compared with the BEST KIDNEY cohort in which RCA was utilized in $<10 \%$ of circuits [9]. Another (yet unpublished) study from France suggested that, in units with low experience with RCA, filter lifespan was not superior to heparin, while the rate of complication was higher. For these reasons, many intensivists throughout the world seem to remain reluctant to introduce RCA in their units as it is seen as complicated, requiring intensive training and might lead to complex metabolic complications [10]. Real-life data, coming from a center with intermediate experience with RCA and outside the context of a randomized trial, are therefore needed.

We therefore decided to retrospectively review data from all CRRT sessions performed in our center during the calendar year 2016, 3 years following the introduction of RCA as a default anticoagulation regimen for CRRT. We sought to evaluate the type and rate of complications associated with RCA compared with systemic heparin anticoagulation during CRRT.

\section{Methods}

\section{Study Design}

This is a monocentric retrospective observational study conducted in the adult intensive care unit (ICU) of the Centre Hospitalier Universitaire Vaudois, a tertiary, teaching hospital located in Lausanne, Switzerland. The ICU contains 35 beds and records approximately 2,000 admissions per year. All consecutive patients admitted to our ICU between January and December 2016 and who received CRRT were included in the study. Patients who declined consent for data reutilization were excluded.

\section{CRRT Initiation}

Our institutional protocol does not specify strict recommendations for CRRT initiation. All therapies were evaluated on a caseby-case basis by treating physician.

\section{CRRT Delivery}

All therapies were delivered using Multifiltrate Pro ${ }^{\circledR}$ CRRT generators (Fresenius Medical Care, Bad Homburg, Germany) and Ultraflux $\mathrm{AV}^{\circledR}$ (Fresenius Medical Care, Bad Homburg, Germany) filters. Therapies were set in a standardized way according to our unit protocol. According to this protocol, RCA represented the default anticoagulation method for CRRT. In patients with contraindications $\mathrm{PT}<40 \%$ in the absence of antivitamin $\mathrm{K}$ therapy (as a marker of severe liver failure), serum lactate level $>4 \mathrm{mmol} / \mathrm{L}$, and need for $>25 \mu \mathrm{g} / \mathrm{min}$ of norepinephrine (as markers of circulatory shock), systemic anticoagulation with heparin was considered. As per the manufacturer's instruction, circuits were electively replaced after $72 \mathrm{~h}$ of running time.

Continuous Veno-Venous Hemodialysis-RCA

CRRT with RCA was delivered in continuous veno-venous hemodialysis (CVVHD) mode with standard solutions $\left(\mathrm{CiCa}^{\circledR} \mathrm{di}\right.$ alysate and $4 \%$ [136 mmol/L] trisodium citrate solution) following an adapted Fresenius ${ }^{\circledR}$ protocol [11]. Dialysate flow rate was adapted according to patients' dry weight aiming for a dose slightly above $25 \mathrm{~mL} / \mathrm{kg} / \mathrm{h}$. Citrate solution flow was started at $4 \mathrm{mmol} / \mathrm{L}$ of blood and titrated according to post-filter ionized calcium measurements. Calcium reinfusion solution consisted in 100 $\mathrm{mmol} / \mathrm{L} \mathrm{CaCl} \mathrm{Cl}_{2}$ solution infused at a rate of $1.7 \mathrm{mmol} / \mathrm{L}$ effluent and titrated according to systemic ionized calcium measurements. Arterial and post-filter blood gases analyses were regularly performed throughout the therapy to monitor $\mathrm{pH}$, base excess, and ionized calcium. Each measurement was reviewed by the physician in charge, and adaptations of blood flow and dialysate were conducted according to our protocol. Total calcium was also monitored throughout the therapy to evaluate citrate accumulation.

Continuous Veno-Venous Hemofiltration-Heparin

For historical and pedagogic considerations, CRRT with systemic heparin anticoagulation was delivered in pre- and post-dilution continuous veno-venous hemofiltration (CVVH) mode with standard substitution solution (Multibic ${ }^{\circledR}$ ). Initial dose was set at $25 \mathrm{~mL} / \mathrm{kg} / \mathrm{h}$ with approximately $33 \%$ of the dose delivered as pre-dilution. Heparin was administered as a continuous infusion via a separate central or peripheral venous line. Heparin infusion was titrated to aim for an anti-Xa activity between 0.3 and $0.6 \mathrm{IU} /$ $\mathrm{mL}$.

In patients with contraindications to both RCA and heparin, a similar protocol was followed but with a lower anti-Xa activity target or no anticoagulation at all according to clinician's judgment.

\section{Data Collection}

Patients' Characteristics and Outcomes

All data were manually collected using electronic chart records (Metavision ${ }^{\circledR}$, IMD Soft, Tel Aviv, Israel) and Soarian ${ }^{\circledR}$ (Cerner, North Kansas City, MO, USA). We collected patients' characteristics on admission as well as ICU and hospital outcomes (survival, length of stay, and dialysis dependence). For the purpose of the study, dialysis dependence was defined as the receipt of any form of renal replacement therapy (RRT) within $72 \mathrm{~h}$ of discharge. For patients with multiple ICU admissions, only the first was considered for baseline data and the last for outcome data. 
Fig. 1. Patients and circuits flow chart. All CRRT circuits were analyzed for lifespan and reason for interruption; however, only CRRT sessions lasting $>6 \mathrm{~h}$ were analyzed for complications and dose delivered. NB: CVVH-Heparin group includes not only circuits ran with therapeutic heparin but also sub-therapeutic and no anticoagulation at all. CRRT, continuous renal replacement therapy; CVVHD-Citrate, continuous veno-venous hemodialysis with regional citrate anticoagulation; CVVH-Heparin, continuous veno-venous hemofiltration with systemic heparin anticoagulation.

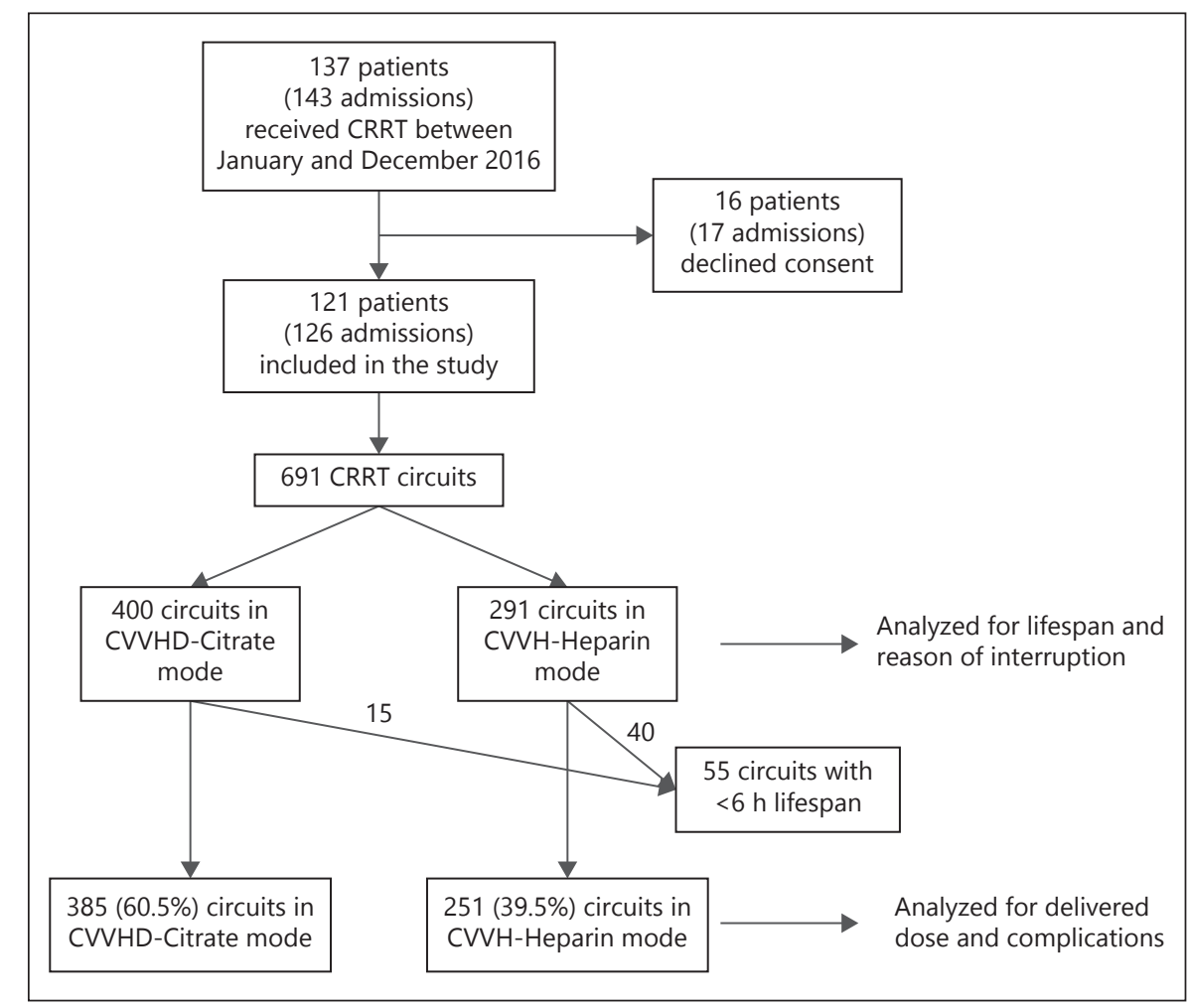

\section{CRRT Sessions}

For all included patients, we then reviewed all consecutive CRRT sessions. A CRRT session was defined as the period of time during which a single CRRT set was connected to a patient. Temporary disconnections were considered as part of a session (recirculation mode). For each session, we have recorded therapy modality (CVVH or CVVHD), anticoagulation method, delivered dose, filter lifespan, and reason for interruption and complications.

\section{Complications}

Electronic medical records were screened for biological alterations occurring during each CRRT session. To assess for complications, we recorded the lowest and highest values of predetermined parameters during a session and the following $12 \mathrm{~h}$. The following predefined complications were recorded: thrombocytopenia (thrombocyte level $<150 \mathrm{G} / \mathrm{L}$ ), hypothermia (body temperature $<35^{\circ} \mathrm{C}$ ), metabolic acidosis ( $\mathrm{pH}<7.35$ and/or BE less than -2$)$, metabolic alkalosis ( $\mathrm{pH}>7.45$ and/or BE $>2$ ), hypocalcemia (serum calcium $<1.05 \mathrm{mmol} / \mathrm{L}$ ), hypernatremia (serum sodium $>145 \mathrm{mmol} / \mathrm{L}$ ), and significant hemorrhage (recorded in medical notes and/or $>2$ red packed cells transfused during sessions). Their severity was determined according to a predetermined scale, presented in the online supplemental Table S1 (for all online suppl. material, see www. karger.com/doi/10.1159/000506253). Alterations present at the beginning of the session (preexisting anomaly) or with an obvious alternate explanation were not considered. For CRRT sessions ran in CVVHD-RCA mode, we also collected changes in blood or dialysate pump flow rate as well as total over ionized calcium ratio and need for calcium substitution to assess citrate intoxication. For this part of the study, only filters with a lifespan longer than $6 \mathrm{~h}$ were considered.

Complications of RCA in CRRT
Delivered Dose

Delivered dose was estimated as the sum of dialysate or preand post-dilution flow rates. For this part of the study, only filters with a lifespan of $6 \mathrm{~h}$ or more were considered.

\section{Statistical Analysis}

Continuous data with normal distribution are reported as mean (SD) and compared using Student $t$ test. Nonnormally distributed data are reported as median (interquartile range [IQR]) and compared using Mann-Whitney $U$ test. Ordinal data are reported as number (percentage) and compared by means of Fisher's exact or chi-square test as appropriate. Kaplan-Meier survival analyses with log-rank test were used to compare circuits' lifespan. A two-sided $p$ value $<0.05$ was considered significant. Prism 8.0.1 and SPSS version 25 were used for statistical analyses.

\section{Results}

\section{Patients' Demographics}

During the study period (Fig. 1), 1,806 patients $(2,011$ admissions) were admitted to our ICU. Of those, 137 (7.6\%) required RRT including 16 who denied institutional consent to participate in research. Hence, $121 \mathrm{pa}-$ tients (126 admissions) were included in the study and analyzed. Their characteristics on ICU admission are presented in Table 1. Briefly, 81 (66.9\%) were males; their median age was 69 years (IQR 11) and median weight 
Table 1. Patients' demographics $(n=121)$ and ICU admissions characteristics $(n=126)$

\begin{tabular}{|c|c|}
\hline \multicolumn{2}{|l|}{ Patients $(\mathrm{n}=121)$} \\
\hline \multicolumn{2}{|l|}{ Demographics } \\
\hline Age, years, median (IQR) & $69(11)$ \\
\hline Body weight, kg, median (IQR) & $79.9(25)$ \\
\hline Gender, male, $n(\%)$ & $81(66.9)$ \\
\hline \multicolumn{2}{|l|}{ Illness severty, median (IQR) } \\
\hline Charlson score & $6(4)$ \\
\hline SAPS II score & $60(21)$ \\
\hline \multicolumn{2}{|l|}{ Renal function parameters, mean (SD) } \\
\hline Baseline creatinine, $\mu \mathrm{mol} / \mathrm{L}$ & $93.5(64.8)$ \\
\hline GFR MDRD, $\mathrm{mL} / \mathrm{min} / 1.72 \mathrm{~m}^{2}$ & $67.9(48.3)$ \\
\hline \multicolumn{2}{|l|}{ Co-existing conditions, $n(\%)$} \\
\hline Chronic kidney injury & $48(39.7)$ \\
\hline HDD & $18(15.1)$ \\
\hline Diabetes mellitus & $39(32.2)$ \\
\hline Chronic hypertension & $74(61.2)$ \\
\hline \multicolumn{2}{|l|}{ Congestive heart failure stage I } \\
\hline$(\mathrm{LVEF}=40-50 \%)$ & $10(8.3)$ \\
\hline \multicolumn{2}{|l|}{ Congestive heart failure stage II } \\
\hline$(\mathrm{LVEF}<40 \%)$ & $20(16.5)$ \\
\hline Peripheral arterial disease & $25(20.7)$ \\
\hline \multicolumn{2}{|l|}{ ICU admissions $(\mathrm{n}=126)$} \\
\hline \multicolumn{2}{|l|}{ Diagnostic at ICU admission, $n(\%)$} \\
\hline Septic shock & $31(24.6)$ \\
\hline Cardiogenic shock & $20(15.9)$ \\
\hline Hemorrhagic shock & $11(8.7)$ \\
\hline Cardiac arrest & $10(7.9)$ \\
\hline AKI & $7(5.6)$ \\
\hline Acute respiratory failure & $6(4.8)$ \\
\hline Intoxication & $2(1.6)$ \\
\hline Others & $39(31)$ \\
\hline \multicolumn{2}{|l|}{ Type of admission (2 missing), $n(\%)$} \\
\hline Medical & $53(44)$ \\
\hline Surgical & $61(50.4)$ \\
\hline Others & $10(8.3)$ \\
\hline \multicolumn{2}{|l|}{ CRRT modality, $n(\%)$} \\
\hline CVVH & $31(24.6)$ \\
\hline CVVHD & $51(40.5)$ \\
\hline CVVH and CVVHD (sequentially) & $44(34.9)$ \\
\hline
\end{tabular}

ICU, intensive care unit; IQR, interquartile range; AKI, acute kidney failure; CRRT, continuous renal replacement therapy; $\mathrm{CVVH}$, continuous veno-venous hemofiltration; CVVHD, continuous veno-venous hemodialysis; HDD, hemodialysis dependancy; SAPS, Simplified Acute Physiology Score; MDRD, modification of diet in renal disease; GFR, glomerular filtration rate; LVEF, left ventricular ejection fraction.

79.9 kg (IQR 25). Median Simplified Acute Physiology Score II was 60 (IQR 21), median Charlson score was 6.0 (IQR 4.0), and 48 (39.7\%) had some degree of preexisting chronic kidney disease, including 18 (15.1\%) who required chronic hemodialysis. The main reason for ICU admission was sepsis (24.6\%) followed by cardiogenic shock (15.9\%) and hemorrhagic shock (8.7\%).

\section{Outcomes}

Among the 121 patients included in the study, 48 (39.7\%) died while in ICU and $10(8.3 \%)$ while on the ward (overall in-hospital mortality 47.9\%). Median ICU length of stay was 8.9 (IQR 15.1) days, and median hospitallength of stay was 23.7 (IQR 41.2) days. Among survivors, 44 (59.5\%) required RRT on ICU discharge (including 16 with preexisting ESRD) and 26 (41.3\%) required RRT on hospital discharge (including 13 with preexisting ESRD).

\section{CRRT Sessions}

Altogether, 691 CRRT sessions were administered to eligible patients for a total duration of $26,055 \mathrm{~h}$ (1,085.6 days). Of those, 400 (57.9\%) were performed in CVVHD-RCA mode and 291 (42.2\%) in CVVH-Heparin mode. Among all considered ICU admissions, both modalities were sequentially administered in 44 (34.9\%) admissions. A single modality was used throughout other admissions, CVVHDRCA in 51 (40.5\%) and CVVH-Heparin in 31 (24.6\%). Patients' characteristics according to CRRT modality are presented in online supplementary Table 2.

\section{Filter Lifespan}

As presented in Figure 2, CVVHD-Citrate mode was associated with a longer filter life compared with CVVHHeparin (median duration 54.9 h [IQR 44.6] vs. $15.3 \mathrm{~h}$ [IQR 22.4], $p<0.0001$ ). This difference remained even when CVVH-Heparin sessions during which $<625 \mathrm{UI} / \mathrm{h}$ of heparin was administered were excluded $(p<0.0001)$ (online suppl. Fig. 1). As shown in Table 2, in CVVHDRCA mode, only $28(8.8 \%)$ of the sessions were interrupted as a result of filter clotting versus $113(42.8 \%)$ in CVVH-heparin mode $(p<0.0001)$. As a consequence, median delivered dose was much higher during sessions in CVVHD-RCA mode: 1.5 (IQR 1.2) L/ kg compared to 0.5 (IQR 0.7) L/kg in sessions in CVVH-Heparin mode $(p<0.0001)$.

\section{Complications}

Complications occurring during CRRT were assessed in 636 CRRT sessions with a duration $>6 \mathrm{~h}$ ( 385 CVVHDRCA, 251 CVVH-Heparin). Main results are presented in Figures 3 and 4 .

\section{Electrolytes}

Hypocalcemia occurred more frequently during CVVHD-RCA sessions compared with CVVH-Heparin sessions: $96(25.07 \%)$ vs. 26 events $(10.79 \%), p<0.0001$. However, this difference disappeared when only moderate and severe hypocalcemia $(<0.95 \mathrm{mmol} / \mathrm{L})$ were con- 
Table 2. CRRT circuit parameters and reasons for interruption comparison between CVVHD-RCA and CVVHHeparin

\begin{tabular}{lccc}
\hline Circuits $(n=691)$ & CVVHD-RCA & CVVH-Heparin & $p$ value \\
\hline RRT characteristics & & & - \\
$\quad$ Total number, $n(\%)$ & $400(57.9)$ & $291(42.2)$ & $<0.0001$ \\
$\quad$ Filter lifespan, h, median (IQR) & $54.9(44.6)$ & $15.3(22.4)$ & $<0.0001$ \\
Dose delivered, mL/kg, median (IQR) & $1.5(1.2)$ & $0.5(0.7)$ & $<0.0002$ \\
Time between ICU admission and & & & $<0.0001$ \\
$\quad$ therapy initiation, h, median (IQR) & $45(77.8)$ & $38.8(59.8)$ & $<0.0001$ \\
Reason for therapy interruption, $n(\%)$ & $28(8.8)$ & $113(43.5)$ & $<0.0001$ \\
Filter clotting & $137(43.2)$ & $64(24.6)$ & $<0.0001$ \\
End of therapy & $134(42.3)$ & $22(8.5)$ & - \\
Time limit of 72 h reached & $18(5.7)$ & $61(23.5)$ & $31(11.7)$ \\
Elevation of transmembrane pressure & $83(20.8)$ & & \\
Missing data & & & \\
\hline
\end{tabular}

NB: CVVH-Heparin group includes not only circuits ran with therapeutic heparin but also sub-therapeutic and no anticoagulation at all.

CRRT, continuous renal replacement therapy; CVVHD, continuous veno-venous hemodialysis; RCA, regional citrate anticoagulation; CVVH-Heparin, continuous veno-venous hemofiltration with systemic heparin anticoagulation; RRT, renal replacement therapy; ICU, intensive care unit; IQR, interquartile range.

Fig. 2. Kaplan-Meier analysis presenting circuit lifespan in CVVHD-Citrate versus CVVH-Heparin methods. Median lifespan was $54.9 \mathrm{~h}$ (IQR 44.6) for CVVHD-RCA versus $15.3 \mathrm{~h}$ (IQR 22.4) for CVVH-Heparin. $p$ value for log-rank test. NB: CVVHHeparin group includes not only circuits ran with therapeutic heparin but also subtherapeutic and no anticoagulation at all. CVVHD, continuous veno-venous hemodialysis; CVVH, continuous veno-venous hemofiltration.

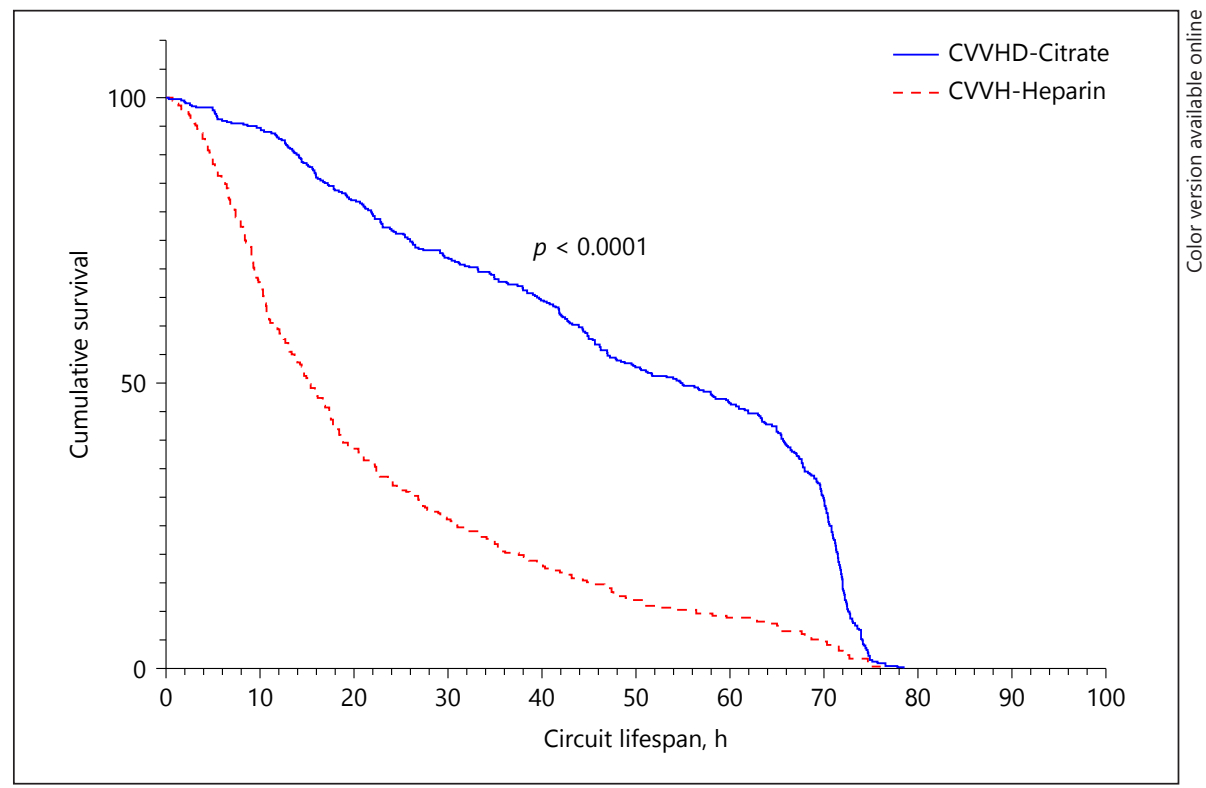

sidered $5(1.3 \%)$ vs. $3(1.2 \%), p=0.99$. There was no difference in the rate of observed hypernatremia between the 2 modalities 13 (3.4\%) vs. $4(2.0 \%), p=0.44$.

\section{Acid-Base Balance}

Overall, metabolic acidosis occurred more frequently during CVVHD-RCA sessions compared with CVVHHeparin sessions $77(20.2 \%)$ vs. 18 (7.2\%), $p<0.0001$. When only severe $(\mathrm{pH}<7.20$ and/or BE less than -6$)$ aci- dosis was considered, no statistically significant difference was observed between the 2 groups 16 (4.2\%) vs. 6 (2.4\%), $p=0.27$.

Similarly, metabolic alkalosis was more frequently observed during CVVHD-RCA sessions than during CVVH-Heparin sessions 187 (48.8\%) vs. 43 (17.1\%), $p=$ 0.0001 . This difference persisted even when only severe $(\mathrm{pH}>7.60$ and/or $\mathrm{BE}>6)$ alterations were considered 54 (14.1\%) vs. $0(0 \%), p<0.0001$. 


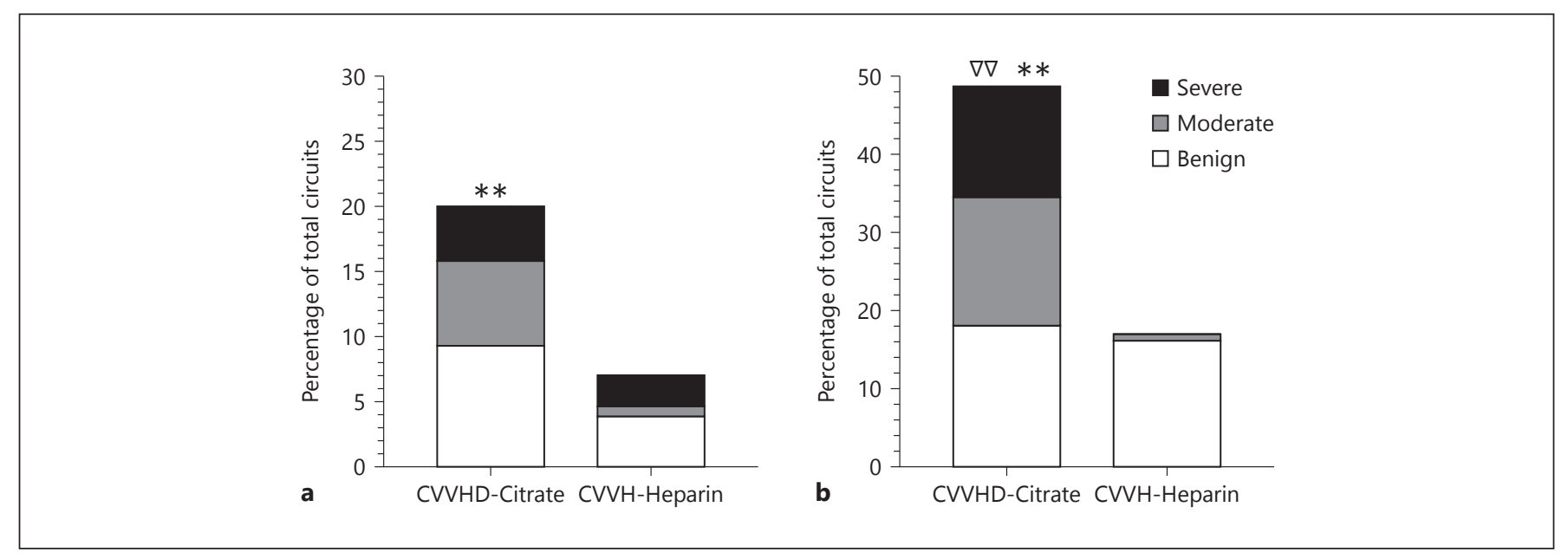

Fig. 3. Therapy-associated acid-base alterations. Complications recorded for CRRT session with a lifespan $>6$ h (385 CVVHDRCA sessions and 251 CVVH-Heparin sessions). a Metabolic acidosis: benign: $\mathrm{pH}<7.35$ and/or $\mathrm{BE}=-2$ : -4 ; moderate: $\mathrm{pH}<7.25$ and/or $\mathrm{BE}=-4:-6$; or severe: $\mathrm{pH}<7.20$ and/or BE less than -6 . $\mathbf{b}$ Metabolic alkalosis: benign: $\mathrm{pH}>7.5$ and/or $\mathrm{BE}=2: 4$; moderate: $\mathrm{pH}>7.55$ and/or $\mathrm{BE}=4: 6$; severe: $\mathrm{pH}>7.6$ and/or $\mathrm{BE}>6$ (severe).

Therapy Adaptations

Blood pump flow was adapted at least once in 53 (13.8\%) CVVHD-RCA circuits and dialysate flow in 38 $(9.9 \%)$.

\section{Citrate Intoxication}

One patient with severe circulatory shock was diagnosed with citrate intoxication although he did not fulfill all criteria (peak total/ionized calcium ratio 2.40). For this patient, therapy was replaced with CVVH-Heparin and the clinical situation eventually improved.

Another patient had a transient elevated total/ionized calcium ratio (peak 2.53) with no other sign of intoxication. Therapy was maintained with parameters normalization over $48 \mathrm{~h}$. This situation was therefore not considered as citrate intoxication.

\section{Hematological Complications}

The incidence of thrombocytopenia was similar during CVVHD-RCA and CVVH-Heparin sessions (6.6 vs. $11.1 \%, p<0.07)$. However, when only severe $(<100 \mathrm{G} / \mathrm{L})$ alterations were considered, there was less thrombocytopenia in therapies ran in CVVHD-RCA group $3(0.8 \%)$ compared to CVVH-Heparin mode 15 (6.4\%), $p<$ 0.0001 .

Clinically significant hemorrhage was observed at a similar rate during CVVHD-RCA and CVVH-Heparin
** $p<0.01$ for overall comparison; ${ }^{\nabla \nabla} p<0.01$ for comparison between severe events. NB: CVVH-Heparin group includes not only circuits ran with therapeutic heparin but also sub-therapeutic and no anticoagulation at all. CVVHD-Citrate, continuous veno-venous hemodialysis with regional citrate anticoagulation; $\mathrm{CVVH}-$ Heparin, continuous veno-venous hemofiltration with systemic heparin anticoagulation.

sessions $23(5.6 \%)$ vs. $11(4.4 \%), p=0.47$. Similarly, there was no difference in terms of number of sessions during which $>2$ packed red blood cells were transfused $35(9.1 \%)$ vs. $22(8.8 \%), p=0.99$.

Heparin-induced thrombocytopenia was diagnosed in 1 patient while undergoing CVVH-Heparin CRRT.

\section{Other Complications}

The rate of documented hypothermia (body temperature $<35^{\circ}$ ) was similar during CVVHD-Citrate and CVVH-Heparin sessions 29 (7.5\%) vs. $22(8.8 \%), p=0.55$.

\section{Discussion}

\section{Summary of Key Findings}

We performed a retrospective observational study on 691 consecutive CRRT sessions to assess the rate and the severity of complications associated with RCA outside the protected setting of randomized controlled trials. We found that, compared with heparin anticoagulation, RCA was associated with an almost fourfold filter lifespan. Such difference was linked to a much lower rate of clotting issues within the circuit. We found that RCA was associated with a higher incidence of electrolyte disorders, in particular acid-base alterations. However, the vast majority of these abnormalities were minor and of unknown 
Fig. 4. Other therapy-associated complications. Complications recorded for CRRT session with a lifespan $>6$ h $(385$ CVVHDRCA sessions and 251 CVVH-Heparin sessions). a Thrombopenia: thrombocyte level: benign: <150 G/L; moderate: $<100 \mathrm{G} / \mathrm{L}$; or severe: $<50$ G/L. b Hypothermia: body temperature: benign: $32-35^{\circ} \mathrm{C}$; moderate: $32-28^{\circ} \mathrm{C}$. c Hypernatremia: sodium plasma level: benign: > $145 \mathrm{mmol} / \mathrm{L}$, moderate: $>152 \mathrm{mmol} / \mathrm{L}$. d Hypocalcemia: systemic ionized calcium: benign: $<1.05 \mathrm{mmol} / \mathrm{L}$; moderate: $<0.95 \mathrm{mmol} / \mathrm{L}$; or severe: $<0.85$ $\mathrm{mmol} / \mathrm{L} .{ }^{* *} p<0.01$ for overall comparison; $\nabla \nabla p<0.01$ for comparison between severe events. NB: CVVH-Heparin group includes not only circuits ran with therapeutic heparin but also sub-therapeutic and no anticoagulation at all. CVVHD-Citrate, continuous veno-venous hemodialysis with regional citrate anticoagulation; CVVH-Heparin, continuous veno-venous hemofiltration with systemic heparin anticoagulation.

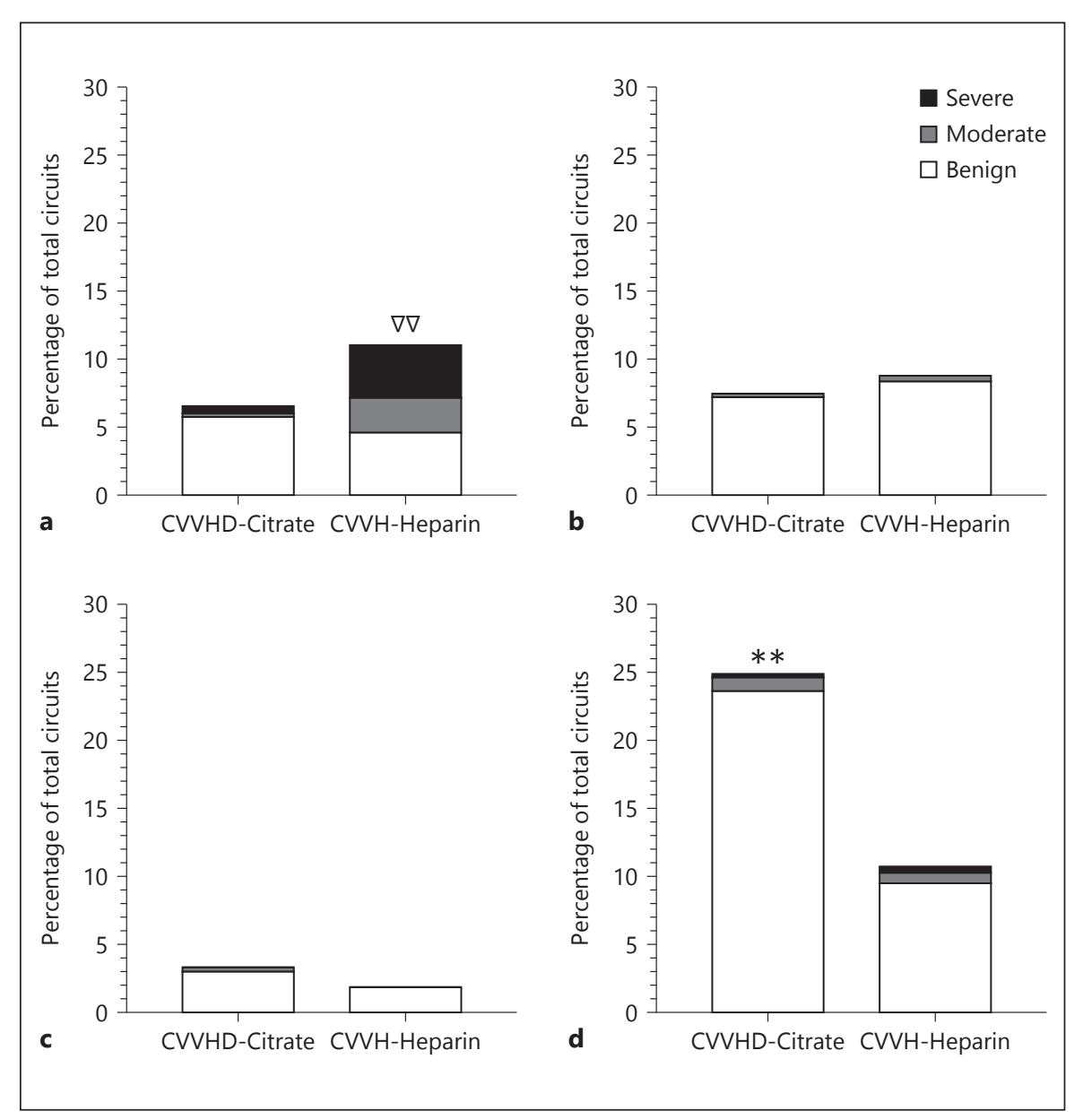

significance. In our selected patients group, only one possible citrate intoxication was observed and no major complication was observed.

\section{Comparison with Previous Studies}

The observed longer filter life and lower rate of circuit clotting associated with RCA are largely consistent with previously reported data $[5,12-16]$. In particular, it is similar to that observed in cohorts using a similar RCA protocol [11]. The short lifespan observed in the heparin group is consistent with previous reports $[12,17]$.

Although we did not perform a cost analysis, our data confirm that RCA introduction should logically translate in important cost savings. In addition, longer filter life translates into lower workload for the nursing staff and improved therapy quality [18].

The reported incidence of metabolic complications associated with RCA is higher than previously reported $[12,14,15]$. This is possibly related to our decision to report any anomaly irrespective of its duration and poten- tial clinical significance. For instance, an isolated abnormal measurement within a 72 -h circuit would be sufficient to be recorded as a complication. The unexpected high rate of benign metabolic acidosis might be related with the relatively high chloride concentration in the dialysate or the composition of the calcium chloride solution. However, when only severe alterations were considered, only metabolic alkalosis was more commonly observed during RCA. In general, metabolic alkalosis during RCA is thought to be benign and easily dealt with [10], although its actual clinical relevance is unknown and cannot be inferred by our data. It might also be related to our team's limited experience at the time of the study. Indeed, as stated in Methods, our RCA protocol prompts a close monitoring of both arterial $\mathrm{pH}$ and calcium levels and suggests therapy adaptation (changes in blood flow or dialysate rate) to normalize these values. Such adaptations were rarely performed and might have prevented the occurrence of some moderate or severe acid-base alterations. Similarly, the observed hypocalce- 
mia could probably have been avoided by more experienced teams.

Several cohorts have reported a lower rate of hemorrhagic complication $[3,4,19-21]$. We have not observed such difference. This is probably related to the very low rate of hemorrhagic complications in our population. For similar reasons, we have not observed a difference in the rate of heparin-induced thrombocytopenia (only 1 case).

\section{Study Implications}

Our data confirm that, even in a center with intermediate experience with RCA, very long circuit lifespan can be achieved. However, the observed higher rate of complications and the fact that the majority of these complications could have been prevented with minor therapy adjustments emphasize the importance of education and experience when RCA is applied. Indeed, we have noted only a small number of therapy adaptations when using RCA, which may be related to staff lacking experience. Implementation of an RCA in a unit should therefore be associated with a strict protocol and intensive education to minimize therapy-associated complications.

\section{Strengths and Limitations}

This study has several strengths. First, our data are based on a large number of observations, close to 700 circuits have been evaluated. Detailed chart review was performed by a single investigator enabling the report of granular data on CRRT practice. Second, we believe that our center is ideal for evaluating RCA complications in real life since it is large $(2,000$ admissions per year) with a large CRRT practice ( $>100$ patients treated per year) but a relatively limited experience with RCA (at the time of the study) and a large number of nursing staff with an important turnover. Hence, our findings might apply to many similar-sized units and perhaps even to smaller ICUs although this remains to be demonstrated.

However, this study also has several limitations worth discussing. First, as a monocentric observational study, result might be associated with bias. There might be limitations in the quality of the data related to nursing/medical documentation. However, complications that were a priori defined would all be identified by routine nursing surveillance in a patient undergoing CRRT. In particular, temperature, arterial blood gas analyses, and electrolyte levels measurement are all part of routine follow-up for such patients in our unit. In addition, our protocol man- dates regular monitoring of systemic and total ionized calcium. All data are automatically entered in our electronic chart records system minimizing the risk of data loss.

Second, the allocation of anticoagulation regimen was far from random since, as per study protocol, RCA was considered to be contraindicated in patients with liver failure (defined as a PT $<40 \%$ ) or circulatory shock (defined as a serum lactate $>4 \mathrm{mmol} / \mathrm{L}$ or need for noradrenaline at a rate $>25 \mu \mathrm{g} / \mathrm{min}$ ). In addition, both modalities were sequentially used in more than a third of the patients. Therefore, no inference on outcomes and mortality can be made from our data. However, this bias is likely to bias results in favor of CVVHD-RCA circuits. Therefore, the higher rate of complication might be under-evaluated.

Third, we have not accounted for the fact that a given complication might have occurred several times in a given patient in different CRRT sessions and we are unable to report on the duration of the abnormalities. However, all these limitations should not bias group comparisons as these apply to both in a similar way.

Fourth, in addition to different anticoagulation modalities, our 2 groups differed also in terms of CRRT modality. CVVH might be associated with shorter filter lifespan compared with CVVHD [22] Indeed, CVVH requires higher blood flows to maintain a low filtration fraction that might in some clinical situations prove difficult. Hence, the difference in filter lifespan between the 2 groups might partially be explained by the difference in modality. However, the influence of CRRT modality on electrolytes or acid-base is less obvious and is unlikely to lead to major bias.

\section{Conclusions}

In a center with intermediate experience, consistent with randomized controlled studies, RCA for CRRT was associated with a much longer circuit lifespan compared with systemic heparin. However, it was associated with a higher rate of mild metabolic complications, in particular metabolic alkalosis. Some of these complications could have been prevented by therapy adaptation. Medical and nursing staff education is of major importance in the implementation of an RCA protocol.

\section{Acknowledgments}

None. 


\section{Statement of Ethics}

The study protocol was approved by the Ethics Committee Vaud (CER-VD 2017-00008). The need for specific individual informed patient consent was waived due to the observational nature of the study. However, patients who declined our institution general consent for data reutilization were excluded from the study.

\section{Disclosure Statement}

N.A.B., M.A., and P.E. stated that they had no conflicts of interest to declare. A.G.S. has received a grant from the Leenaards foundation, speaker honoraria from Fresenius Medical Care, and consulting honoraria from B. Braun Melsungen AG.

\section{Funding Sources}

The study was funded by the ICU research fund.

\section{Author Contributions}

N.A.B. carried out data collection, participated in data interpretation and analyses, and drafted the manuscript. M.A. and P.E. participated in study design and critically reviewed the manuscript. A.G.S. participated in study design, supervised data collection, participated in data interpretation and analyses, and critically reviewed the manuscript. All authors read and approved the final manuscript and agree to be personally accountable for their contribution.

\section{References}

1 KDIGO. Kidney Disease: Improving Global Outcomes (KDIGO) Acute Kidney Injury Work Group (2012) KDIGO clinical practice guidelines AKI: AKI definition. Kidney Int. 2012;2(1):19-36

2 Gattas DJ, Rajbhandari D, Bradford C, Buhr H, Lo S, Bellomo R. A Randomized Controlled Trial of Regional Citrate Versus Regional Heparin Anticoagulation for Continuous Renal Replacement Therapy in Critically Ill Adults. Crit Care Med. 2015 Aug;43(8): $1622-9$.

3 Schilder L, Nurmohamed SA, Bosch FH, Purmer IM, den Boer SS, Kleppe CG, et al.; CASH study group. Citrate anticoagulation versus systemic heparinisation in continuous venovenous hemofiltration in critically ill patients with acute kidney injury: a multi-center randomized clinical trial. Crit Care. 2014 Aug; 18(4):472.

4 Stucker F, Ponte B, Tataw J, Martin PY, Wozniak H, Pugin J, et al. Efficacy and safety of citrate-based anticoagulation compared to heparin in patients with acute kidney injury requiring continuous renal replacement therapy: a randomized controlled trial. Crit Care. 2015 Mar;19(1):91.

5 Bai M, Zhou M, He L, Ma F, Li Y, Yu Y, et al. Citrate versus heparin anticoagulation for continuous renal replacement therapy: an updated meta-analysis of RCTs. Intensive Care Med. 2015 Dec;41(12):2098-110.

6 Khadzhynov D, Schelter C, Lieker I, Mika A, Staeck O, Neumayer $\mathrm{HH}$, et al. Incidence and outcome of metabolic disarrangements consistent with citrate accumulation in critically ill patients undergoing continuous venovenous hemodialysis with regional citrate anticoagulation. J Crit Care. 2014 Apr;29(2):265-71.

7 Zhang W, Bai M, Yu Y, Li L, Zhao L, Sun S, et al. Safety and efficacy of regional citrate anticoagulation for continuous renal replacement therapy in liver failure patients: a systematic review and meta-analysis. Crit Care. 2019 Jan; 23(1):22.
8 Garzotto F, Ostermann M, Martín-Langerwerf D, Sánchez-Sánchez M, Teng J, Robert R, et al.; DoReMIFA study group. The Dose Response Multicentre Investigation on Fluid Assessment (DoReMIFA) in critically ill patients. Crit Care. 2016 Jun;20(1):196.

9 Uchino S, Bellomo R, Morimatsu H, Morgera S, Schetz M, Tan I, et al. Continuous renal replacement therapy: a worldwide practice survey. The beginning and ending supportive therapy for the kidney (B.E.S.T. kidney) investigators. Intensive Care Med. 2007 Sep;33(9): 1563-70.

10 Schneider AG, Journois D, Rimmelé T. Complications of regional citrate anticoagulation: accumulation or overload? Crit Care. 2017 Nov;21(1):281.

11 Morgera S, Schneider M, Slowinski T, VargasHein O, Zuckermann-Becker $\mathrm{H}$, Peters $\mathrm{H}$, et al. A safe citrate anticoagulation protocol with variable treatment efficacy and excellent control of the acid-base status. Crit Care Med. 2009 Jun;37(6):2018-24.

12 Borg R, Ugboma D, Walker DM, Partridge R. Evaluating the safety and efficacy of regional citrate compared to systemic heparin as anticoagulation for continuous renal replacement therapy in critically ill patients: A service evaluation following a change in practice. J Intensive Care Soc. 2017 Aug; 18(3): 184-92.

13 Chowdhury SR, Lawton T, Akram A, Collin $\mathrm{R}$, Beck J. Citrate versus non-citrate anticoagulation in continuous renal replacement therapy: results following a change in local critical care protocol. J Intensive Care Soc. 2017 Feb;18(1):47-51.

14 Gutierrez-Bernays D, Ostwald M, Anstey C, Campbell V. Transition From Heparin to Citrate Anticoagulation for Continuous Renal Replacement Therapy: Safety, Efficiency, and Cost. Ther Apher Dial. 2016 Feb;20(1): 53-9.

15 Huguet M, Rodas L, Blasco M, Quintana LF, Mercadal J, Ortiz-Pérez JT, et al. Clinical im- pact of regional citrate anticoagulation in continuous renal replacement therapy in critically ill patients. Int J Artif Organs. 2017 Nov; 40(12):676-82.

$16 \mathrm{Wu}$ MY, Hsu YH, Bai CH, Lin YF, Wu CH, Tam KW. Regional citrate versus heparin anticoagulation for continuous renal replacement therapy: a meta-analysis of randomized controlled trials. Am J Kidney Dis. 2012 Jun; 59(6):810-8.

17 Park JS, Kim GH, Kang CM, Lee CH. Regional anticoagulation with citrate is superior to systemic anticoagulation with heparin in critically ill patients undergoing continuous venovenous hemodiafiltration. Korean J Intern Med (Korean Assoc Intern Med). 2011 Mar; 26(1):68-75.

18 Rewa OG, Eurich DT, Noel Gibney RT, Bagshaw SM. A modified Delphi process to identify, rank and prioritize quality indicators for continuous renal replacement therapy (CRRT) care in critically ill patients. J Crit Care. 2018 Oct:47:145-52.

19 Brandenburger T, Dimski T, Slowinski T, Kindgen-Milles D. Renal replacement therapy and anticoagulation. Best Pract Res Clin Anaesthesiol. 2017 Sep;31(3):387-401.

20 Hetzel GR, Schmitz M, Wissing H, Ries W, Schott G, Heering PJ, et al. Regional citrate versus systemic heparin for anticoagulation in critically ill patients on continuous venovenous haemofiltration: a prospective randomized multicentre trial. Nephrol Dial Transplant. 2011 Jan;26(1):232-9.

21 Monchi M, Berghmans D, Ledoux D, Canivet JL, Dubois B, Damas P. Citrate vs. heparin for anticoagulation in continuous venovenous hemofiltration: a prospective randomized study. Intensive Care Med. 2004 Feb;30(2): 260-5.

22 Ricci Z, Ronco C, Bachetoni A, D'amico G, Rossi S, Alessandri E, et al. Solute removal during continuous renal replacement therapy in critically ill patients: convection versus diffusion. Crit Care. 2006;10(2):R67. 